\title{
Enhanced Recovery Program after Colorectal Surgery
}

\author{
Magdy Mahmoud Mostafa, MD; Mohamed Hassan Elkaser, MD; Hatem Ahmed Elsayed*, M.B.B.Ch
}

*Corresponding author:

Hatem Ahmed Elsayed,

E-mail: dr.hatemhatem@gmail.com Received for publication January31, 2020;

Accepted February,9, 2020;

Published on line February 23, 2020.

Copyright 2020 The Authors published by Al-Azhar University, Faculty of Medicine, Cairo, Egypt. All rights reserved. This an openaccess article distributed under the legal terms, where it is permissible to download and share the work provided it is properly cited. The work cannot be changed in anyway or used commercially.

doi: 10.21608/aimj.2020.23737.1140

General Surgery Department, Faculty of Medicine, Al-Azhar University, Cairo, Egypt

\begin{abstract}
Background: Patients undergoing major gastro-intestinal tract surgery are at risk of nutritional depletion due to surgical stress, inadequate nutritional intake and subsequent increase in metabolic rate. The aim of this work is to evaluate the outcome of early oral feeding, balanced analgesia and enforced mobilization, which are integral parts of fast track rehabilitation program of colorectal surgery, versus delayed oral feeding and regular forms of mobilization and pain control in patients undergoing colorectal surgery.
\end{abstract}

Patients and methods: This study was a prospective randomized clinical trial included 40 patient's undergoing colorectal surgery.

Results: Group II showed a high statistically significant operation time than Group I. $(\mathrm{p}<0.001)$. Group II showed a high statistically significant NPO while Group I showed a high Oral liquid diet, Full liquid diet and Regular diet $(\mathrm{p}<0.001)$.

Conclusion: The application of an enhanced recovery program for "elective" colorectal surgery has consistently been shown to decrease postoperative complication, shorten length of hospital stay, facilitate patient recovery, shorten convalescence, and lower healthcare cost.

Keywords: Recovery Program; Stomach; Pain control; Colorectal Surgery.

Disclosure: The authors have no financial interest to declare in relation to the content of this article. The Article Processing Charge was paid for by the authors. Authorship: All authors have a substantial contributions to the article

\section{INTRODUCTION}

Enteral feeding when compared with total parental nutrition may prevent gastrointestinal tract (GIT) mucosal atrophy, attenuate the trauma stress response, maintain immunecompetence and preserve normal gut flora ${ }^{1}$. A period of starvation (nil by mouth) is a common practice after intestinal anastomosis. The stomach is decompressed with nasogastric tube and IV fluids are given with oral feeding being introduced after GIT dysmotility is being resolved ${ }^{2}$.

The rational of nil by mouth is to prevent post-operative nausea and vomiting and to protect anastomosis by allowing it time to heal before being stressed by food. It is however unclear whether deferral of enteral feeding is beneficial ${ }^{3}$.Fast-track surgery (FTS) is a relatively new set of protocols that has been implemented in a number of hospitals to enhance recovery after surgery 4 . By reducing both the physiological and psychological stresses associated with operations and minimizing pain and discomfort, proponents of FTS argue that, there is less organ dysfunction and hence, a reduction in the need for hospitalization, with earlier hospital discharge ${ }^{4}$. Proponents of FTS believe that the surgical stress response is no longer necessary to restore homeostasis as modern peri-operative management has advanced to provide adequate fluid, temperature and glycemic support if necessary 5 .
To date, twenty recognized protocols make up the Fast-track program ${ }^{6}$. FTS combines various techniques used in care of the patients including optimal pain control, and aggressive post-operative rehabilitation, including early enteral nutrition and ambulation. The combination of these approaches reduced the stress response organ dysfunction and therefore greatly shorten the time required for full recovery ${ }^{7}$. Postoperative pain control, ambulation and complete recovery of the gut and urinary bladder function are essential prerequisites in planning discharge from hospital ${ }^{8}$.

The aim of this work was to evaluate the outcome of early oral feeding, balanced analgesia and enforced mobilization, which were integral parts of fast track rehabilitation program of colorectal surgery, versus delayed oral feeding and regular forms of mobilization and pain control in patients undergoing colorectal surgery. 


\section{PATIENTS AND METHODS}

These study was prospective randomized clinical trial included 40 patients underwent colorectal surgery for main etiology for both benign and malignant conditions, whom admitted to the general surgery department, Al-Azhar university hospitals.

The patients were randomly allocated into two groups. Randomization was achieved using sealed envelopes. After carefully explaining the purpose of the study, informed consent was obtained from every patient.

Colorectal pathology managed by surgical resection with age ranged from 20-75 years and possible curative respectability are included in the study .

Elderly >75 years, uncompensated cardiopulmonary disease, Immunological disorders such as Systemic Lupus Erythematosus; sarcoidosis and patients on immunosuppressive therapy for any cause were excluded from the study.

Study randomized into 2 groups; Group 1 included 20 patients that underwent colorectal surgeries including different types of anastomosis, either handmade or stapler assisted. They were submitted to Fast track protocol. Preoperative pre-admission counseling gave; a clear explanation of what was to happen during hospitalization, explanation of the role of the patient about food intake, mobilization after surgery. Fasting was 2 hours for liquid and 6 hours for solids. Pre-operative nutrition and good carbohydrate diet was achieved in each patient. Venous thromboembolism prophylaxis and antibiotic prophylaxis were given.

Intraoperative fluid therapy: Combination of both crystalloid and colloid with vasopressor, keep intraoperative fluid therapy less than $3000-3500 \mathrm{ml}$ and no routine use of drains or nasogastric tube.

Postoperative analgesia, early oral intake, early mobilization and early removal of nasogastric tube, drains and catheters if any.

Patients in this group started (were allowed) clear fluids as soon as they are awake, a full liquid diet on first postoperative day, and a normal diet on the second postoperative day as tolerated (indicated by an absence of vomiting or abdominal distension).

The patients were given information on the importance of early mobilization and were encouraged to stay out of bed for 4 hours on the first postoperative day, 6 hours on the following day, and 8 hours on subsequent days. Further, they were asked to walk the length of the ward, $2 \times 30$ meters, once on postoperative day 1 , twice on day 2 , and three times on subsequent days.

Postoperative analgesia:In these patients, an epidural catheter was inserted at the low thoracic region (e.g. T8-9, T9-10, T10-11 spaces) and continuous infusion of $2 \mathrm{mg} / \mathrm{ml}$ of Levobupivacaine and $2 \mathrm{ug} / \mathrm{ml}$ of Fentanyl, at a rate of 4 to $8 \mathrm{ml} / \mathrm{h}$, was established. On the following morning, the concentration of Levo-bupivacaine was reduced to $1.5 \mathrm{mg} / \mathrm{ml}$ using a portable infusion pump. Infusion was continued until the morning of postoperative day 2 and during this period the patient was admitted to intermediate care unit for the care of epidural catheter.

Group 2 (conventional rehabilitation program): included 20 patients who were managed in the traditional way.

Diet: Nothing by mouth for 5 days until the resolution of ileus, then a fluid diet, followed by a regular diet.
Mobilization: Patients were mobilized by nurses on demand and will receive regular instructions about the importance of mobilization. Mobilization was recorded by patients assisted by nursing staff in the same manner as for intervention group.

Postoperative analgesia: Pain was controlled by giving 10 $\mathrm{mg} / \mathrm{ml}$ paracetamol infusion to the maximum $500 \mathrm{mg}$ paracetamol infusion bottles every 6 hours and ketorolac 30 $\mathrm{mg}$ ampoule by IV infusion in $100 \mathrm{ml}$ normal saline solution every 12 hours.

All patients included in this study were subjected to: Complete history taking, clinical examination, laboratory investigation, the necessary radiological investigation, all cases were properly prepared by correction of electrolyte and acid-base disturbance and all cases were undergoing colonic preparation either elective or emergency.

Post-operative assessment including: Clinical assessment for occurrence of early post-operative complications; surgical cardiovascular or pulmonary complications.

Statistical analysis: Recorded data were analyzed using the statistical package for social sciences, version 20.0 (SPSS Inc., Chicago, Illinois, USA). Quantitative data were expressed as mean \pm standard deviation (SD). Qualitative data were expressed as frequency and percentage. The following tests were done: Independent-samples t-test of significance was used when comparing between two means. Chi-square $\left(\mathrm{x}^{2}\right)$ test of significance was used in order to compare proportions between two qualitative parameters. The confidence interval was set to $95 \%$ and the margin of error accepted was set to $5 \%$. The p-value was considered significant as the following: Probability (P-value): P-value $<0.05$ was considered significant. P-value $<0.001$ was considered as highly significant. $\mathrm{P}$-value $>0.05$ was considered insignificant

\section{RESULTS}

Demographic data has found Gender: Group I consisted of 12 males $(60.0 \%)$ and 8 female $(40.0 \%)$, while Group II consisted of 13 males $(65.0 \%)$ and 7 females $(35.0 \%)$, with a statistically non-significant difference in gender distribution between groups $(\mathrm{p}=0.744)$. Age: The mean age in Group I was $55.85 \pm 11.91$ years in comparison to $57.60 \pm 9.29$ years in Group II, with statistically non-significant difference between groups $(\mathrm{p}=0.607)$. Weight: The mean Weight in Group I80.55 $\pm 10.12 \mathrm{~kg}$ in comparison to $88.25 \pm 8.25 \mathrm{~kg}$ in Group II, with a statistically significant difference between groups $\left(\mathrm{p}=0.012^{*}\right)$. Height: The mean Height in Group $\mathrm{I} 171.60 \pm 8.28 \mathrm{~cm}$ in comparison to $8173.10 \pm 5.34 \mathrm{~cm}$ in Group II, with a statistically non-significant difference between groups $(p=0.500)$. Smoker, Marital status, and ABO system: the difference was a statistically non-significant between groups $(\mathrm{p}=1.000)$.

Comparison between the studied groups according to operation time showed that; .t:Student t-test, p:pvalue for comparing between the studied groups. Statistically significant at $\mathrm{p} \leq 0.05$ (table 1 ). 


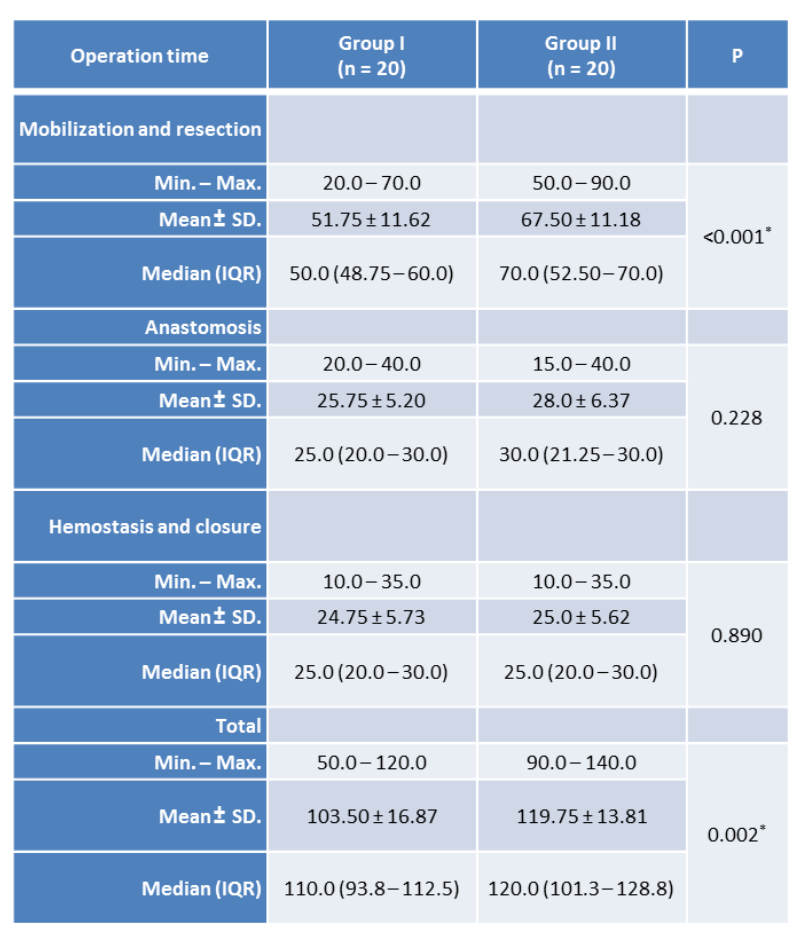

Table 1: Comparison between the studied groups according to operation time: Mobilization and resection: Group II showed a high statistically significant Mobilization and resection than Group I. $\left(\mathrm{p}<0.001^{*}\right)$. Anastomosis: Group I and Group II showed the same anastomosis. $(\mathrm{p}=0.228)$.Hemostasis and closure: Group I and Group II showed the same Hemostasis and closure. $(\mathrm{p}=0.228)$. Total: Group II showed a high statistically significant operation time than Group I. $\left(\mathrm{p}<0.001^{*}\right)$

Comparison between the studied groups according to nutrition From Day 0 to Day 4 showed that; Group II showed a high statistically significant NPO while Group I showed a high Oral liquid diet, Full liquid diet and Regular diet $\left(\mathrm{p}<0.001^{*}\right)$.

\section{DISCUSSION}

This study reports the initial results of our experience with an enhanced recovery program in a 40 patients with mean age in Group I was $55.85 \pm 11.91$ years in comparison to $57.60 \pm$ 9.29 years in Group II undergoing elective colonic and rectal surgery $(85.0 \%$ of patients in Group I and $90.0 \%$ Group II was prone to Elective preparation. We offered the Enhanced recovery program to patients undergoing colorectal surgery for main etiology for both benign and malignant conditions (25.0\% of patients in Group I and $20.0 \%$ Group II was Benign. $75.0 \%$ of patients in Group I and $80.0 \%$ Group II was Malignant), whom admitted to the general surgery department, Al-Azhar university Hospital.

Pelvic drains in $80.0 \%$ of Group I and in 94.1 of Group II. The difference between groups was statistically nonsignificant $(\mathrm{p}=.1 .000)$.according to Eleven Randomized controlled trials included 1803 patients in total (939 patients in the drain group and 864 patients in the no drain group). Meta-analysis showed that there were no statistically significant differences between the drain group and the no drain group in overall anastomotic leakage, clinical anastomotic leakage, radiologic anastomotic leakage, mortality, wound infection, re-operation and respiratory complications ${ }^{9}$.

Rollins et al. ${ }^{10}$ reported that Comparison between the studied groups according to colonic preparation: $85.0 \%$ of patients in Group I and $90.0 \%$ Group II was prone to Elective preparation. The difference between groups was a statistically non-significant $\left(p^{=} 1.000\right)$.The use of mechanical bowel preparation does not affect the incidence of postoperative complications when compared with no preparation. Hence, mechanical bowel preparation should not be administered routinely prior to elective colorectal surgery.

Group I (ERP) showed a high Oral liquid fluid at day of operation, a high oral liquid diet at day 1 , full liquid diet and Regular diet after day 2. The difference was a statistically significant $\left(\mathrm{p}<0.001^{*}\right)$. There have been several studies looking at the issue of early feeding after abdominal surgery. A meta-analysis was published in 2001 looking at early feeding versus a restricted diet. Eleven studies concluded that there was no benefit in adhering to a restricted diet ${ }^{11}$. They pointed out that post laparotomy disturbed motility predominantly affects the stomach. The small bowel and colon recovers normal function between 4 and 8 hours, with feeding tolerated and food absorbed within 24 hours. Although early feeding was associated with an increased risk of vomiting, the meta-analysis did not appear to find any other clear advantage in keeping patients nil by mouth after gastrointestinal resection. Early normalization of gastrointestinal motility after open colonic resection was started with a multimodal rehabilitation program involving early oral feeding, mobilization and laxative treatment. Early oral feeding within 24 hours after gastrointestinal surgery is well tolerated, safe and plays an important role to enhance recovery and outcome ${ }^{12}$.

Group I Walking a significant long distance than group II till day $5\left(\mathrm{p}<0.001^{*}\right.$. Prolonged immobility is associated with a host of complications including thromboembolic disease, skeletal muscle loss and weakness, atelectasis, and insulin resistance ${ }^{\mathbf{1 3}}$. In most observational studies, adherence with early and progressive mobilization targets is a significant predictor of earlier discharge ${ }^{\mathbf{1 4}}$. Patients in enhanced recovery programs meet mobilization targets sooner. It is important to emphasize to patients the importance of an early mobilization plan with daily goals for time out of bed and distance walked beginning as soon as the day of surgery.

In the present study, at day 1: Group I stay out of bed $3.70 \pm$ 0.66 hours than group II $0.55 \pm 0.76$ hours. At day 2: Group I stay out of bed $5.45 \pm 0.89$ hours than group II $0.55 \pm 0.76$ hours. At day 3: Group I stay out of bed $7.50 \pm 1.24$ hours than group II $1.85 \pm 0.88$ hours. At day 4 : Group I stay out of bed $7.95 \pm 0.22$ hours than group II $2.35 \pm 1.09$ hours. At day 5: Group I stay out of bed $8.0 \pm 0.0$ hours than group II 2.95 \pm 1 .10hours. Group I stay out of bed a long significant hours than group II till day $5\left(\mathrm{p}<0.001^{*}\right)$. While convincing evidence suggests that patients should not be kept in bed after surgery, there is little guidance on how best to achieve early mobilization, particularly whether adhering to a specific structured mobilization protocol has additional benefits compared to allowing patients to mobilize at will (i.e. as tolerated).One challenge in performing trials of complex interventions is the selection of the primary outcome. As the negative effects of bed rest are well-known (e.g. thromboembolism, pneumonia, muscle wasting and physical deconditioning), mobilization could be hypothesized to decrease the risk of complications associated with immobilization.

Contemporary colorectal surgery is often associated with long length of stay ( 8 days for open surgery and 5 days for laparoscopic surgery), high $\operatorname{cost}^{22}$ and rates of surgical site infection approaching $20 \%$. ${ }^{15}$. During the hospital stay for elective colorectal surgery, the incidence of perioperative nausea and vomiting (PONV) may be as high as $80 \%$ in 
patients with certain risk factors ${ }^{\mathbf{1 6}}$. After discharge from colorectal surgery, readmission rates have been noted as high as $35.4 \% .6^{17}$. Our results can be compared to those in the recent literature where a good Enhanced recovery program adherence is associated with both a reduction of length of hospital stay (LOS) and improvements in surgical outcomes ${ }^{18}$. Group II stayed in hospital a significant long Duration than group I $(4.15 \pm 1.66$ days $)\left(\mathrm{p}<0.001^{*}\right)$.

\section{CONCLUSION}

The application of an enhanced recovery program for "elective" colorectal surgery has consistently been shown to decrease postoperative complication. It has also shorten length of hospital stay and lower healthcare cost.

\section{REFERENCES}

1. Akbaba S, Kayaalp C and Savkilioglu M. Nasogastric decompression after total gastrectomy. Hepatogastroenterology, 2004; 51:1881-5.

2. Scott NA, Susnerwala S and Levine E. Preoperative neo-adjuvant therapy for curable rectal cancer-reaching a consensus 2008. Colorectal Dis., 2009; 11(3):245-8.

3. Smith FM, Waldron D and Winter DC. Rectumconserving surgery in the era of chemoradiotherapy. Br J Surg., 2010; 97(12):1752-64.

4. Kehlet H, and Wilmore DW. Fast-track surgery. Br J Surg., 2005; 92: 3-4.

5. Holte K, and Kehlet H. Epidural anaesthesia and analgesia e effects on surgical stress responses and implications for postoperative nutrition. Clin Nutr., 2002; 21:199e206.

6. Lassen K, Soop M and Spies C. Consensus review of optimal perioperative care in colorectal surgery: Enhanced Recovery After Surgery (ERAS) Group recommendations. Arch Surg., 2009; 144:961-9.

7. Basse L, Hjort JD and Billesbolle P. A clinical pathway to accelerate recovery after colonic resection. Ann Surg., 2000; 232:51-7.

8. Kahokehr AA, Sammour $\mathrm{T}$ and Sahakian V. Influences on length of stay in an Enhanced Recovery
Program in colonic surgery. Colorectal Dis., 2010; 2010:9999.

9. Zhang PJ, Siegel R and DeSantis C, et al. Colorectal cancer statistics, 2014. Cancer Journal for Clinicians, 2014; 64(2): 104-117.

10. Rollins KE, Javanmard-Emamghissi $\mathrm{H}$ and Lobo DN. Impact of mechanical bowel preparation in elective colorectal surgery: A meta-analysis. World $J$ Gastroenterol., 2018; 24(4):519-536.

11. Andersen HK, Lewis SJ and Thomas S. Early enteral nutrition within $24 \mathrm{~h}$ of colorectal surgery versus later commencement of feeding for postoperative complications. Cochrane Database Syst Rev., 2006; CD 004080.

12. Brower RG. Consequences of bed rest. Crit Care Med., 2009; 10: 422-8.

13. Vlug MS, Bartels SA, Wind J, et al. Which fast track elements predict early recovery after colon cancer surgery. Colorectal Dis., 2012; 14 (8): 1001-8.

14. Slieker J, Frauche P, Jurt J, et al. Enhanced recovery ERAS for elderly: a safe and beneficial pathway in colorectal surgery. Int J Colorectal Dis., 2017; 32:215-21.

15. Kang CY, Chaudhry OO, Halabi WJ, et al. Outcomes of laparoscopic colorectal surgery: data from the Nationwide Inpatient Sample 2009. Am J Surg., 2012; 204:952-957.

16. Thiele RH, Rea KM, Turrentine FE, et al. Standardization of care: impact of an enhanced recovery protocol on length of stay, complications, and direct costs after colorectal surgery. J Am Coll Surg., 2015; 220:430-443.

17. Eberhart LH, Mauch M, Morin AM, et al. Impact of a multimodal anti-emetic prophylaxis on patient satisfaction in high-risk patients for postoperative nausea and vomiting. Anaesthesia., 2002; 57:10221027.

18. Nagle D, Pare T, Keenan E, et al. Ileostomy pathway virtually eliminates readmissions for dehydration in new ostomates. Dis Colon Rectum., 2012; 55:12661272 . 